\title{
Lso-HPE1, an Effector of 'Candidatus Liberibacter solanacearum', Can Repress Plant Immune Response
}

\author{
Julien G. Levy,1,† Rachel Gross, ${ }^{4}$ Azucena Mendoza-Herrera, ${ }^{2}$ Xiaotian Tang, ${ }^{2}$ Kevin Babilonia, ${ }^{3}$ Libo Shan, ${ }^{3}$ \\ Joseph C. Kuhl, ${ }^{4}$ Margaret S. Dibble, ${ }^{4}$ Fangming Xiao, ${ }^{4}$ and Cecilia Tamborindeguy ${ }^{2, \dagger}$ \\ ${ }^{1}$ Department of Horticultural Sciences, Texas A\&M University, College Station, TX 77843 \\ ${ }^{2}$ Department of Entomology, Texas A\&M University, College Station, TX 77843 \\ ${ }^{3}$ Institute for Plant Genomics and Biotechnology, Department of Plant Pathology and Microbiology, Texas A\&M University, College Station, \\ TX 77843 \\ ${ }^{4}$ Department of Plant Sciences, University of Idaho, Moscow, ID 83844 \\ Accepted for publication 3 November 2019.
}

\begin{abstract}
'Candidatus Liberibacter solanacearum' is a plant pathogen affecting the families Solanaceae and Apiaceae in different parts of the world. ' $C a$. L. solanacearum' is a Gram-negative, fastidious $\alpha$-proteobacterium that is vectored by different psyllid species. Plant-pathogenic bacteria are known for interfering with the host physiology or defense mechanisms, often by secreting bacterial effectors. Effector proteins are critical for virulence; therefore, the identification of effectors could help with disease management. In this study, we characterized the Sec-translocondependent ' $\mathrm{Ca}$. L. solanacearum'-hypothetical protein effector 1 (LsoHPE1). We compared this protein sequence in the different ' $C a$. L. solanacearum' haplotypes. We predicted the signal peptide and validated its function using Escherichia coli's alkaline phosphatase fusion assay.
\end{abstract}

ABSTRACT
Agrobacterium tumefaciens-mediated transient expression in Nicotiana benthamiana demonstrated that Lso-HPE1 from ' $\mathrm{Ca}$. L. solanacearum' haplotypes A and B were able to inhibit the induction of cell death in plants. We also compared gene expression of the Lso-HPE1- transcripts in ' $\mathrm{Ca}$. L. solanacearum' haplotypes A and B in tomato and in the vector Bactericera cockerelli. This work validates the identification of a Sectranslocon-dependent ' $\mathrm{Ca}$. L. solanacearum' protein possibly involved in suppression of plant cell death.

Keywords: analytical and theoretical plant pathology, Bactericera cockerelli, bacteriology, 'Candidatus Liberibacter solanacearum', Lso haplotype, potato, psyllid, tomato, zebra chips
'Candidatus Liberibacter solanacearum' is a fastidious Gramnegative bacterium that infects different plant families worldwide. Several ' $\mathrm{Ca}$. L. solanacearum' haplotypes have been identified infecting solanaceous or apiaceous crops (Glynn et al. 2012; Lin et al. 2012; Nelson et al. 2011). The ' $\mathrm{Ca}$. L. solanacearum' A and B haplotypes (LsoA and LsoB) are transmitted by the tomato psyllid Bactericera cockerelli (Hemiptera: Triozidae), which is also known as the potato psyllid. These haplotypes infect solanaceous plants in North and Central America and New Zealand (Liefting et al. 2008, 2009), causing damage and increasing costs to potato and tomato production. Last year, the existence of LsoF-infected potato was reported in the United States; however, it has not been determined yet whether this is a psyllid-borne pathogen (Swisher Grimm and Garczynski 2019). In contrast, LsoC, LsoD, and LsoE are transmitted by carrot psyllids and infect apiaceous crops in the Europe and the Mediterranean Basin (Alfaro-Fernández et al. 2012; Tahzima et al. 2014; Teresani et al. 2014). Presently, no commercially acceptable host plant resistance has been identified against any Liberibacter pathogen. Therefore, the main strategy to

†Corresponding authors: J. Levy: julienlevy@tamu.edu; and C. Tamborindeguy ctamborindeguy@tamu.edu

Funding: This project is supported by the Texas A\&M AgriLife Research Insect Vector Disease Grant Program: Controlling exotic and invasive insect transmitted pathogen. This project is supported by the United States Department of Agriculture, National Institute of Food Agriculture, Agriculture and Food Research Initiative competitive award number 2017-67013-2656.

*The $e$-Xtra logo stands for "electronic extra" and indicates that three supplementary figures are published online.

The author(s) declare no conflict of interest.

(c) 2020 The American Phytopathological Society control these pathogens relies on calendar applications of pesticides. This strategy imposes an additional cost to the industries affected by Liberibacter pathogens. Indeed, for example, the cost associated with psyllid control in the Pacific Northwest region of the United States amounts to $\$ 11$ million per year (Greenway and Rondon 2018). Hence, novel approaches to control these pathogens are urgently needed.

Although ' $C a$. L. solanacearum' can complete its life cycle in both eukaryotic hosts (the plant and the vector), it is presently unculturable, which makes performing ' $\mathrm{Ca}$. L. solanacearum' studies difficult. However, the genomes of several ' $C a$. L. solanacearum' haplotypes are available in NCBI (https:// www.ncbi.nlm.nih.gov/genome/genomes/2244). Those include three LsoA, two LsoB, one LsoC, and one LsoD strain sequences which can help in identifying the molecular basis of ' $\mathrm{Ca}$. L. solanacearum'-host interactions. Nevertheless the genetic determinants that control the host range of different ' $\mathrm{Ca}$. L. solanacearum' haplotypes in both plants and insect hosts remain unknown.

Differences between ' $C a$. L. solanacearum' haplotypes have been reported in the interaction with plants and vectors (Tamborindeguy et al. 2017). In potato, infection with LsoA or LsoB causes zebra chip (Crosslin et al. 2010; Secor and Rivera-Varas 2004). This disease results in diverse aerial symptoms common to other pathologies. Additionally, tubers from infected plants show a characteristic striped pattern that intensifies after frying (Munyaneza et al. 2007). Both LsoA and LsoB haplotypes cause similar symptoms, albeit with differences, and, in both cases, the infection of potato plants results in early plant death (Harrison et al. 2019; Swisher Grimm et al. 2018). An incidental and small sample of LsoC-infected potato that did not show any symptoms was reported in Finland (Haapalainen et al. 2018). In tomato, ' $\mathrm{Ca}$. L. solanacearum' infection induces spiky apical leaves, chlorosis and 
mottling of leaves, plant stunting, and fruit deformation (Liefting et al. 2009; Rojas-Martinez et al. 2016). Thus, LsoA and LsoB haplotypes represent a valuable system to decipher the molecular basis of ' $C a$. L. solanacearum' pathogenicity because they are transmitted by the same vector and infect the same plant species but present differences in virulence. For instance, LsoB infection causes early death of tomato plants approximately 8 weeks after infection whereas LsoA-infected tomato plants continue to live and can produce fruit as long as they are properly maintained (MendozaHerrera et al. 2018).

Bacterial effectors play an important role in promoting pathogenicity during the interaction of diverse plant-pathogen systems (Macho and Zipfel 2015). Bacterial effectors are proteins secreted by pathogens and delivered into the cells of their hosts. These proteins often interfere with the host physiological or immune responses for the benefit of the pathogen to invade or survive in the host (Toruño et al. 2016). Microorganisms that depend on other hosts to complete their life cycle manipulate their hosts to permit colonization. For example, these microorganisms can release effectors to modulate the physiological response of their eukaryotic host; this could be through direct or indirect suppression of the host defense response. To counteract infections, likely as a consequence of evolution, some plant species further evolve specific immune receptors to recognize these effectors to trigger immune responses. In this case, the effectors function as avirulence factors and trigger effector-triggered immunity (ETI) upon recognition by plant resistance $(\mathrm{R})$ proteins (Jones and Dangl 2006). One major group of $\mathrm{R}$ proteins are the nucleotide-binding site leucine-rich repeat (NBS-LRR) type, which are similar to the family of mammalian nucleotide-binding oligomerization domainLRR proteins that function in immune responses as well (DeYoung and Innes 2006). In plants, $R$ proteins can trigger programmed cell death as a defense response, also known as hypersensitive response (HR), upon recognition of pathogen avirulence effectors. Although the mechanism of $\mathrm{R}$ protein activation is not completely understood, it is believed that the $\mathrm{R}$ protein undergoes a conformational change to switch from an inactive state to an active state which can then lead to programmed cell death to prevent the pathogen from spreading in the host (Du et al. 2012). Effector discovery and later characterization of the effector functions and host targets are pivotal to the development of disease resistance strategies.

' $\mathrm{Ca}$. L. solanacearum' is related closely to ' $\mathrm{Ca}$. Liberibacter' spp. responsible for huanglongbing, also known as citrus greening disease. These include 'Ca. L. asiaticus', ' $C a$. L. africanus', and ' $C a$. L. americanus'. Whereas, in 'Ca. L. asiaticus', several effectors have been identified and tested for different functions (Clark et al. 2018; Jain et al. 2018; Pitino et al. 2016), only ' $\mathrm{Ca}$. L. solanacearum' serralysin has been reported as a potential effector (Ravindran et al. 2018).

In this study, we focused on a ' $C a$. L. solanacearum' gene encoding a hypothetical protein that was identified as a putative secreted protein based on bioinformatic analyses (Prasad et al. 2016). First, we compared the amino acid sequence of the encoded protein among the different ' $\mathrm{Ca}$. L. solanacearum' haplotypes and Liberibacter genomes. Second, we evaluated the expression of the transcripts in LsoA- and LsoB-infected tomato plants and psyllids. Third, we tested whether the ' $\mathrm{C} a$. L. solanacearum' protein encoded a functional secretion signal peptide (SP). Finally, we evaluated whether the protein could act as an effector that interferes with plant defenses. To do this, we tested the ability of the protein to suppress plant defense mechanisms such as Prf ${ }^{\mathrm{D} 1416 \mathrm{~V}_{-}}$or Bax-induced cell death response in Nicotiana benthamiana using a transient expression system. In tomato, the NBS-LRR resistance protein Prf can induce cell death upon recognition of pathogen avirulence effectors (Salmeron et al. 1996). A Prf variant with a single amino acid substitution, Prf ${ }^{\mathrm{D} 1416 \mathrm{~V}}$, mimics the effector-induced active state of the protein and causes bacteria- or effector-independent programmed cell death when transiently expressed in leaves of
N. benthamiana (Du et al. 2012). This system has been developed as a tool to test effectors. Similarly, Bax is a mammalian protein that functions as a regulator of apoptosis and can cause cell death and accumulation of the defense-related protein PR1 when expressed in $N$. benthamiana (Lacomme and Santa Cruz 1999). This system can also be used to evaluate the function of putative effectors (Xiang et al. 2016).

\section{MATERIALS AND METHODS}

Plant material. Tomato (Solanum lycopersicum) (Moneymaker) and $N$. benthamiana seed were grown in Sunshine Mix (Sun Gro Horticulture Canada) on light shelves under a cycle of $16 \mathrm{~h}$ of light and $8 \mathrm{~h}$ of darkness at 21 to $24^{\circ} \mathrm{C}$.

'Ca. L. solanacearum'-hypothetical protein effector 1 sequence validation. $B$. cockerelli colonies harboring LsoA or LsoB (Yao et al. 2016) were maintained on tomato plants as was previously described (Ibanez et al. 2017). DNA from pools of 10 insects from each colony was purified using the cetyltrimethylammonium bromide method (Nachappa et al. 2011).

The 'Ca. L. solanacearum'-hypothetical protein effector 1 (LsoHPE1) gene sequence (ADR52633.1, formerly known as CKC_ 04420) was amplified by PCR using DNA from each colony. Briefly, $50 \mathrm{ng}$ of DNA were combined with $0.2 \mu \mathrm{l}$ of Phusion HighFidelity DNA Polymerase (New England Biolabs, Ipswich, MA), $4 \mu \mathrm{l}$ of $5 \times$ Phusion HF Buffer, $0.4 \mu \mathrm{l}$ of $10 \mathrm{mM}$ dNTPs, $1 \mu \mathrm{l}$ of each primer at $10 \mathrm{nM}$ (Table 1), and molecular-grade water. The PCR conditions were $98^{\circ} \mathrm{C}$ for $30 \mathrm{~s}$; followed by 35 cycles of $98^{\circ} \mathrm{C}$ for $10 \mathrm{~s}, 55^{\circ} \mathrm{C}$ for $30 \mathrm{~s}$, and $72^{\circ} \mathrm{C}$ for $30 \mathrm{~s}$; and a final extension at $72^{\circ} \mathrm{C}$ for $6 \mathrm{~min}$. The amplicons were purified using a PureLink PCR Purification Kit (Invitrogen, Carlsbad, CA) and ligated into a pGEM-T easy vector using the pGEM-T Easy cloning kit (Promega Corp., Madison, WI). The ligation was transformed into One Shot TOP10 competent cells (Invitrogen). Transformed colonies were selected and plasmid DNA extractions from at least three colonies were performed using a PureLink Quick Plasmid Miniprep Kit (Invitrogen) and sequenced by Eton Bioscience Inc. (San Diego, $\mathrm{CA}$ ). The obtained sequences were compared with the ' $\mathrm{Ca}$. L. solanacearum'-sequenced genomes.

Protein sequence analysis. Bioinformatic analysis of the LsoHPE1 protein sequence to predict SPs was performed using SignalP version 5.0 (Almagro Armenteros et al. 2019) with default settings for Gram-negative bacteria. A Blastp search analysis was performed to identify the most similar proteins in other Liberibacter spp.

Phylogenetic analysis. For the phylogenetic analysis, sequences were aligned with ClustalW using the MEGA 5.2 software (Tamura et al. 2011). The phylogenetic reconstruction was done by Bayesian inference using MrBayes 3.2 (Ronquist et al. 2012). Markov-chain Monte Carlo runs were carried out for 1,000,000 generations, and the first $25 \%$ of sampled trees were discarded as burn-in. Tree information was visualized and edited using FigTree v1.4.3 (http://tree.bio.ed.ac.uk/software/figtree). The ' $\mathrm{Ca}$. L. asiaticus' protein identified by Blast analysis (WP_012778427) was used as outgroup.

Alkaline phosphatase assays. The 78 nucleotide sequence coding for the putative SP plus 15 additional nucleotides encoding 5 extra amino acids of the Lso-HPE1 protein was amplified by PCR. The PCR product was purified on a column using the QIAquick kit (Qiagen, Valencia, CA), digested with the SacI and MfeI restriction enzymes (NEB), and cloned into a previously digested pJDT-SDM1 vector using T4 DNA ligase (NEB) to obtain an in-frame gene fusion with the gene $p h o A$ in the vector. One Shot TOP10 competent cells were transformed with the resulting construct. The transformed bacteria were selected on Lysogeny broth (LB) agar plates containing ampicillin at $100 \mu \mathrm{g} / \mathrm{ml}$. The insert was verified by PCR and sequencing. The transformed bacteria were tested for alkaline phosphatase (PhoA) activity as previously published by 
Ammerman et al. (2008) as follows. Bacteria were grown on LB agar plates containing ampicillin at $100 \mu \mathrm{g} / \mathrm{ml}, 5$-bromo-4-chloro3 -indolyl phosphate (5-BCIP) at $90 \mu \mathrm{g} / \mathrm{ml}$ as chromogenic substrate, and $75 \mathrm{mM} \mathrm{Na}_{2} \mathrm{HPO}_{4}$ to block endogenous phosphatase activity. Escherichia coli bacteria transformed with the empty plasmid pJTD-SDM1 were used as negative control. We also verified the secretion using the overnight liquid culture of LsoHPE1-phoA and the pJTD1 empty vector. For that, $1.5 \mathrm{ml}$ of liquid culture was centrifuged for $15 \mathrm{~min}$ at 3,000 rpm. The supernatants were recovered, filtered through a $0.2-\mu \mathrm{m}$ filter (VWR), and incubated with 5-BCIP at $90 \mu \mathrm{g} / \mathrm{ml}$ at room temperature for $2 \mathrm{~h}$ (Supplementary Fig. S1). We also spotted the filtered supernatants on LB-ampicillin plates and verified that no bacterium grew after an overnight incubation at $37^{\circ} \mathrm{C}$, showing that the supernatants were free of bacterial contamination.

Cloning of Lso-HPE1 into pEarlyGate 101 and Agrobacterium transformation. Lso-HPE1 from psyllids harboring LsoA was amplified as previously described using the Phusion High-Fidelity DNA Polymerase. Lso-HPE1 from psyllids harboring LsoB was amplified using Platinum Taq Polymerase (Thermo Fisher Scientific, Waltham, MA). Primers used are described in Table 1 . The amplicons were cloned in the pENTR vector by TOPO cloning according to manufacturer's directions (Thermo Fisher Scientific) and verified by sequencing. The purified $\mathrm{pENTR}$ vector containing Lso-HPE1 was digested with $M l u I$, and the insert was then transferred to pEarlyGate 101 (pEG101) with the GateWay LR Clonase II enzyme (Thermo Fisher Scientific) following the manufacturer's recommendations and verified by sequencing. The Agrobacterium tumefaciens strain LBA4404 was transformed following the heat shock method.

A. tumefaciens-mediated transient expression. The Agrobacterium-mediated transient expression assays were performed as follows. All bacteria were grown for $24 \mathrm{~h}$ at $28^{\circ} \mathrm{C}$ and $280 \mathrm{rpm}$ in $3 \mathrm{ml}$ of LB supplemented with kanamycin $(50 \mu \mathrm{g} / \mathrm{ml}), 10 \mathrm{mM}$ morpholineethanesulfonic acid (MES), and $20 \mu \mathrm{M}$ acetosyringone. Cells were pelleted and resuspended in fresh buffer containing $10 \mathrm{mM}$ MES, $200 \mu \mathrm{M}$ acetosyringone, and $10 \mathrm{mM} \mathrm{MgCl}_{2}$. Cells were resuspended at an optical density at $600 \mathrm{~nm}\left(\mathrm{OD}_{600}\right)=0.7$, except those containing the $\operatorname{Prf}^{\mathrm{D} 1416 \mathrm{~V}}$, which were resuspended at $\mathrm{OD}_{600}=0.3$. Agrobacterium suspensions were infiltrated into 5-week-old $N$. benthamiana leaves using 2-ml disposable syringes. Agrobacterium suspensions were either infiltrated alone or in coinfiltration. Western blot analyses were conducted to verify LsoHPE1 protein expression (Supplementary Figs. S1, S2, and S3). After infiltration, plants were maintained on light shelves at room temperature $\left(21\right.$ to $\left.24^{\circ} \mathrm{C}\right)$. We repeated each experiment three times and included six to eight plants per experiment. We infiltrated six to eight leaves per plant. Cell death was scored at variable time points following infiltration, usually at 4,6 , and 8 days postinfiltration. To evaluate the ability of Lso-HPE1 to disrupt cell death, coinfiltrations were performed with pCXSN-Bax and PrfD1416V separately. As a control, pCXSN-Bax and Prf ${ }^{\mathrm{D} 1416 \mathrm{~V}}$ were coinfiltrated with pEG101 empty vector (negative control) and pBETX-AvrPto, separately. The cell death response was scored on a scale of 0 (no reaction) to 1 (cell death); a score of 0.5 was used if partial cell death reaction occurred; that is, when spots of cell death developed in the infiltrated area (Supplementary Fig. S2). The average of cell death per treatment was calculated and is reported.

Gene expression analysis. B. cockerelli colonies carrying either LsoA or LsoB were maintained and tested in our laboratory as previously described (Nachappa et al. 2014; Yao et al. 2016). Ten ' $C a$. L. solanacearum'-infected insects from each colony were pooled. Insect RNA samples were extracted and processed as previously published (Ibanez et al. 2014). Total RNA from each pool of insects was purified using RNeasy Mini kit (Qiagen) followed by DNase treatment with RNase-Free DNase (Qiagen). Three pools per colony were analyzed.

LsoA- and LsoB-infected plant samples were collected from plants previously infested with LsoA- or LsoB-infected insects. Leaf tissue was collected from the top-most fully expanded leaf of each plant 3 weeks postinfection and immediately flash frozen in liquid nitrogen. After the leaf sample was collected, each plant was kept until the end of the experiment in order to collect plant tissue for DNA extraction to verify ' $\mathrm{Ca}$. L. solanacearum' presence or absence. ' $C a$. L. solanacearum' in plants can be detected 4 weeks after B. cockerelli infestation (Levy et al. 2011). The flash-frozen tissue samples were ground to fine powder. Total RNA extraction was performed using the RNeasy Plant Mini Kit (Qiagen) according to the manufacturer's instructions. The RNA samples were treated with RNase-Free DNase (Qiagen).

To quantify gene expression in plants and insects, quantitative PCR (qPCR) was performed following previously published methods (Ibanez et al. 2014). Total RNA (50 ng) was reverse transcribed into cDNA and the qPCR was performed in the same reaction using the Luna Universal one-step RT-qPCR kit (NEB), following the manufacturer's protocol. The amplification protocol consisted of one step at $55^{\circ} \mathrm{C}$ for $10 \mathrm{~min}$ (reverse transcription), one step at $95^{\circ} \mathrm{C}$ (initial denaturation), and 40 cycles of $95^{\circ} \mathrm{C}$ for $15 \mathrm{~s}$ and $60^{\circ} \mathrm{C}$ for $30 \mathrm{~s}$ using a QuantStudio 6 Flex Real-Time PCR System (Thermo Fisher Scientific). Each reaction contained $100 \mathrm{ng}$ of RNA, $0.25 \mu \mathrm{M}$ each primer (Table 1), $5 \mu \mathrm{l}$ of Luna Universal OneStep Reaction Mix (2×), $0.5 \mu$ l of Luna WarmStart RT Enzyme Mix $(20 \times)$, and molecular-grade water. Reactions for all samples were performed in duplicate, with negative controls in each run. The mean threshold cycle $(\mathrm{Ct})$ values and standard deviation were calculated. The Rec A (ADR52764.1, CKC_05085) primers were used as bacterial housekeeping gene control (Ibanez et al. 2014). $\Delta \mathrm{Ct}$ was calculated for the average of three biological replicates for each condition. Mean and standard error of the mean were calculated and reported. Quantitative PCR analyses were analyzed using one-way analysis of variance (ANOVA) with Tukey's posthoc test in R. Levene's test was carried out to test for equality of variances and the assumption was met.

The accession numbers for the different sequences are as follows: LsoA = WP_045960716, KJZ81751.1(NZ1), and KQC48998 $(\mathrm{RSTM}) ; \mathrm{LsoC}=$ WP_076969720, 112aa FIN111, ONI58619.1, and

TABLE 1. Primers used in the study

\begin{tabular}{lll}
\hline Primer name & \multicolumn{1}{c}{ Sequence 5'-3' } & Comments \\
\hline Lso-HPE1F $(\mathrm{SacI})$ & CACGAGCTCATGAATTTTTTAATGTTAGC & Cloning in PJDT1 \\
Lso-HPE1R $(\mathrm{Mfe}$ I) & CACCAATTGTATTTCATAAACTGACTGTG & Cloning in PJDT1 \\
PJDT1-F & CAGGAAACAGCTATGAC & Sequencing of PJDT1 \\
PJDT1-R & CGCTAAGAGAATCACGCAGAGC & Sequencing of PJDT1 \\
Gene Lso-HPE1 F & AATGGCGGGAGAGTATTAAA & Sequencing of Lso-HPE1 \\
Gene Lso-HPE1 R & AATATGAAAAATCCATCGGAT & Sequencing of Lso-HPE1 \\
qPCR-F & TGTCAAATGTCTAAGTTGCGAATTG & Expression of Lso-HPE1 \\
qPCR-R & ATCGGTCATGACTATGGCATTT & Expression of Lso-HPE1 \\
Gatway Lso-HPE1F (LsoA and LsoB) & CACCATGAATTTTTAATGTTAGC & Gateway cloning Lso-HPE1 \\
Gatway Lso-HPE1R (LsoA) & GTGACGCGCAGAATCATTA & Gateway cloning Lso-HPE1LsoA \\
Gatway Lso-HPE1F (LsoB) & CTGATGCGCAGAATCCTTA & Gateway cloning Lso-HPE1LsoB \\
\hline
\end{tabular}


FIN114 ONI59537; LsoD1 = RPD37081; and 'Ca. L. asiaticus' = WP_012778427.

\section{RESULTS}

Alignment and phylogenetic analysis. A search of LsoHPE1 homologs using Blast analyses identified one homolog in each ' $C a$. L. solanacearum' genome, except in the strains Henne A and R1 (NCBI reference sequences NZ_JQIG00000000.1 and NZ_ JNVH00000000.1, respectively). The identity among ' $\mathrm{Ca}$. L. solanacearum' Lso-HPE1 proteins was over $86 \%$, and the proteins ranged from 111 to 119 amino acids long. Of note, there is a conserved domain between LsoA and LsoB proteins in the Cterminal region of the proteins which was not conserved among the other ' $C a$. L. solanacearum' proteins (Fig. 1, last 12 amino acids). A 98-amino-acid-long putative homolog was also identified in ' $\mathrm{Ca}$. L. asiaticus' (WP_012778427), albeit with a lower degree of similarity $(43.68 \%$ identity and $\mathrm{E}$ value $=5 \mathrm{e}-13)$. No similar proteins were identified in ' $C a$. L. africanus', ' $C a$. L. americanus', ' $C a$. L. europaeus', or Liberibacter crescens. All ' $\mathrm{Ca}$. L. solanacearum' proteins clustered together in the phylogenetic tree (Fig. 2).

SP prediction and validation. SignalP-5.0 predicted an SP with a cleavage site between amino acids 20 and 21 (GKA-QS; probability: 0.7018). This is in agreement with the in silico analysis that identified Lso-HPE1 as a Sec-dependent presecretory protein with a putative SP (Prasad et al. 2016). The SP of Lso-HPE1 is conserved in all ' $\mathrm{Ca}$. L. solanacearum' proteins and is $100 \%$ identical in each haplotype (Fig. 1).

Analysis of the Lso-HPE1 SP revealed the presence of a hydrophobic core, as expected for SEC targeting signals (Cranford-Smith and Huber 2018). However, this core is not flanked by a positively charged $\mathrm{N}$ domain, located $\mathrm{N}$ terminal to the hydrophobic core, and a less positively charged $\mathrm{C}$ domain, as are other SEC targeting signals (Cranford-Smith and Huber 2018).

Therefore, to validate the presence of an active SP in Lso-HPE1, we tested the motif in $E$. coli using the $p h o A$ gene fusion assay. Although the control E. coli cells with an empty pJDT1-SDM vector remained white, the bacteria with the fusion Lso-HPE1 SP-phoA turned blue after a 6-h incubation (Fig. 3). We confirmed the function of the SP by testing the phosphatase activity using a filtered supernatant from a bacterial liquid culture (Supplementary Figure S1). This validated the activity of the Lso-HPE1 SP.
Suppression of plant cell death. We tested whether LsoHPE1 from LsoA and LsoB were able to reduce Prf ${ }^{\mathrm{D} 1416 \mathrm{~V}_{\text {-induced }}}$ cell death in three independent experiments. The coinfiltration of

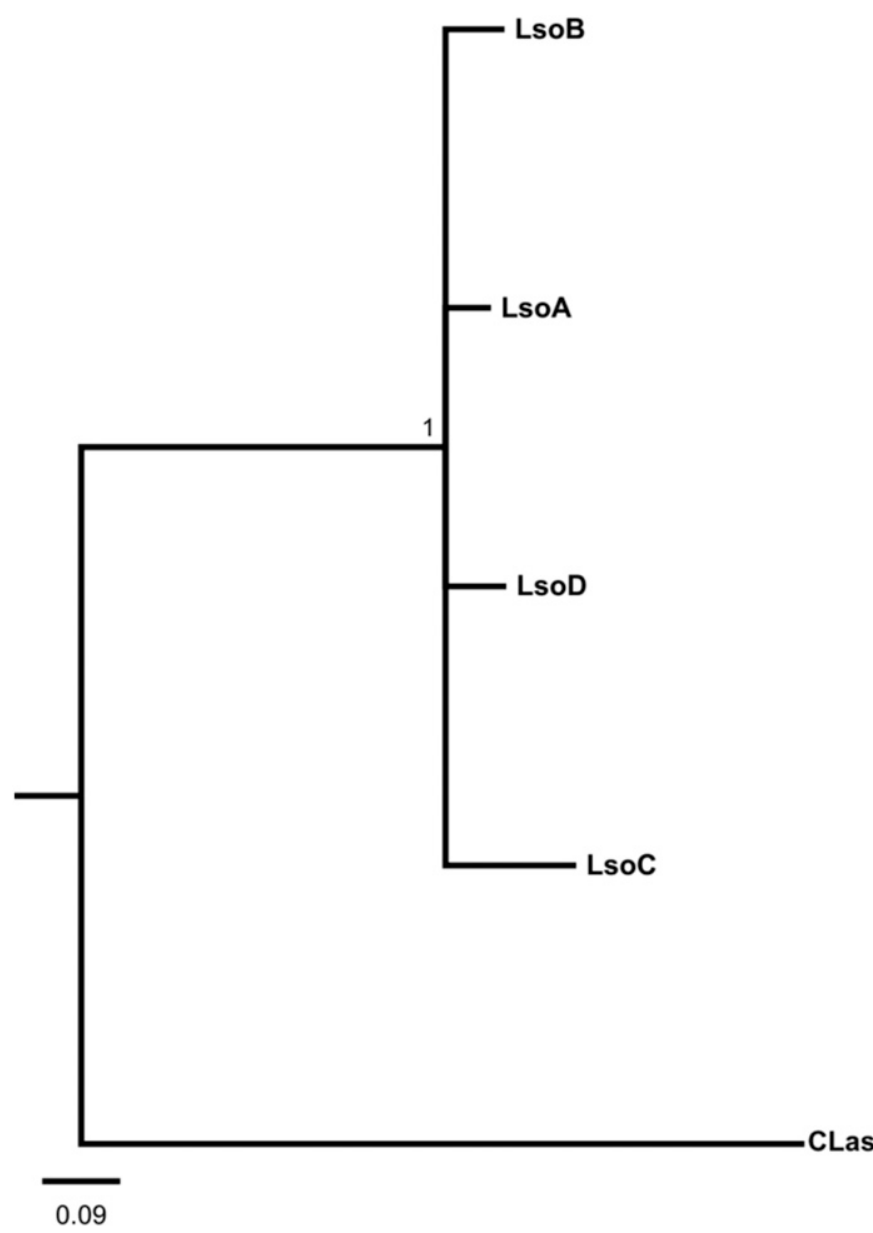

Fig. 2. Phylogenetic analysis of Liberibacter homologs to 'Candidatus Liberibacter solanacearum' (Lso)-hypothetical protein effector 1 protein under Bayesian inference. Scale bar indicates branch length. The ' $\mathrm{Ca}$. L. asiaticus' (CLas) protein was used as outgroup. Numbers at the nodes denote posterior probabilities (support values $>0.7$ are shown).

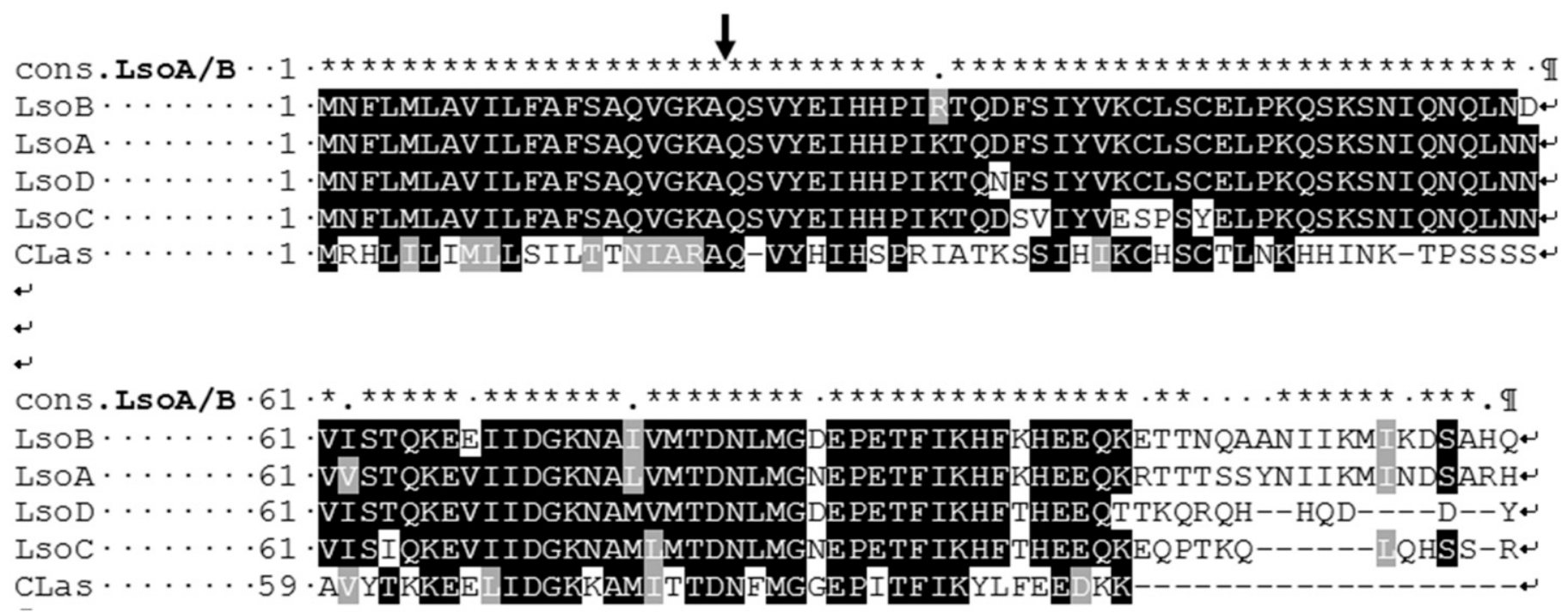

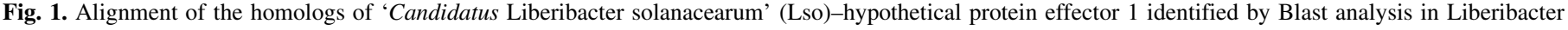

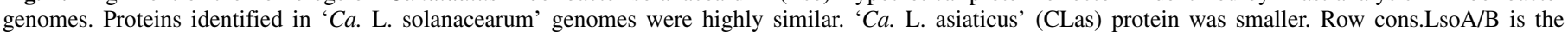

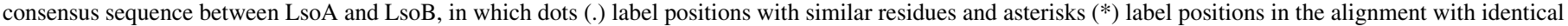
residues. The arrow between amino acids 20 and 21(GKA-QS) marks the putative cleavage site of the signal peptide. 

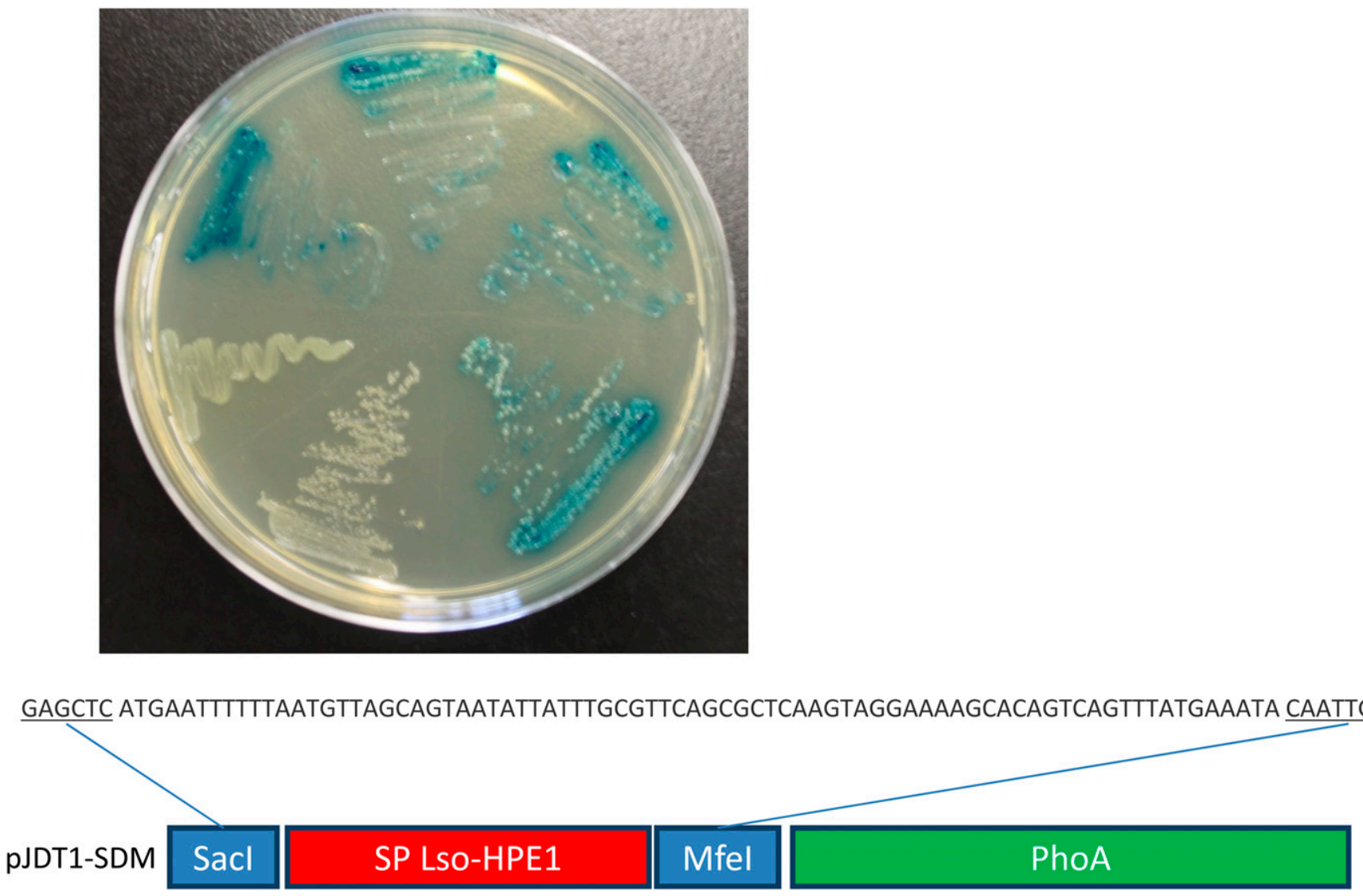

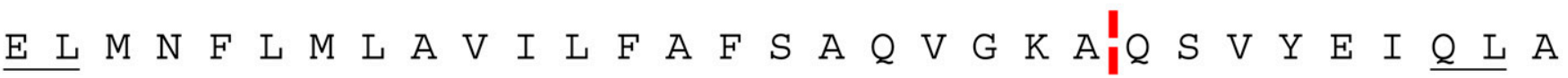

Fig. 3. Alkaline phosphatase (PhoA) assay validated the prediction of the signal peptide of 'Candidatus Liberibacter solanacearum'-hypothetical protein effector 1 (Lso-HPE1). The top panel shows the results of the assay: four independent Escherichia coli clones with the fusion Lso-HPE1-phoA changed color while two independent clones without the signal peptide (SP) remained white when grown on Lysogeny broth containing 5-bromo-4-chloro-3-indolyl phosphate. The bottom panel is a diagram of the fusion of the Lso-HPE1 SP and the phoA gene. The vertical line in the amino acid sequence represents the predicted cleavage site in the Lso-HPE1 protein. Underlined in the nucleic acid and the protein sequences are the restriction sites introduced for the cloning.

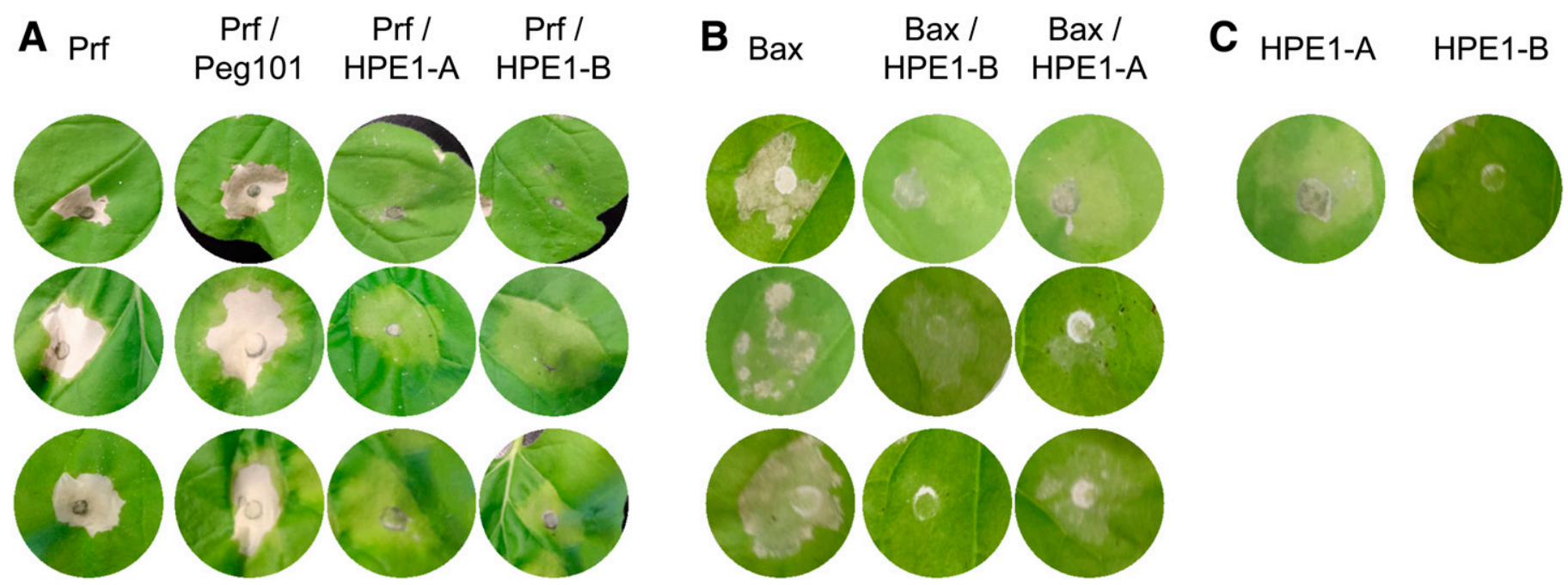

Fig. 4. Cell death induction after Agrobacterium infiltration. Pictures were taken 7 days postinfiltration. The picture shows the cell death phenotype 7 days after infiltration. Each column is labeled with infiltration construct, in the following order: A, PrfD1416V alone (Prf) showed cell death (score 1), coinfiltration of PrfD1416V-Peg101 empty vector showed cell death (score 1), coinfiltration of PrfD1416V and 'Candidatus Liberibacter solanacearum'-hypothetical protein effector 1 (Lso-HPE1)-A did not show cell death (score 0), and coinfiltration of PrfD1416V-Lso-HPE1-B did not show cell death (score 0). B, Bax alone showed cell death (score 1), coinfiltration of Bax-Lso-HPE1-A and coinfiltration of Bax-Lso-HPE1-B did not show cell death (score 0). C, Infiltration of Lso-HPE1-A and Lso-HPE1-B alone did not show cell death (score 0). 
Prf ${ }^{\mathrm{D} 1416 V}$ and Lso-HPE1 from LsoA or from LsoB resulted in a reduction of cell death development. Although the average cell death score was 0.92 when $\operatorname{Prf}^{\mathrm{D} 1416 \mathrm{~V}}$ was infiltrated alone, coinfiltration of PrfD1416V with Lso-HPE1 from LsoA or with Lso-HPE1 from LsoB scored, on average, 0.36 and 0.26, respectively (Fig. 4A). As a positive control, $\operatorname{Prf}^{\mathrm{D} 1416 \mathrm{~V}}$ was coinfiltrated with AvrPto and scored, on average, 0.15 in the cell death assays, whereas coinfiltration of pEG101 empty vector with Prf D1416V scored, on average, 0.85.

Similarly, coinfiltration of Bax and Lso-HPE1 from LsoA and $\mathrm{LsoB}$ resulted in reduction of cell death. In this case, the average cell death score was 0.8 for Bax alone whereas the coinfiltrations of Bax with Lso-HPE1 from LsoA or LsoHPE1 from LsoB scored, on average, 0.33 and 0.36 , respectively (Fig. 4B).
The infiltration of Lso-HPE1 from LsoA or from LsoB alone (Fig. 4C) did not induce cell death.

Gene expression. Lso-HPE1 expression was compared between LsoA- and LsoB-infected plants and between LsoA- and LsoB-infected psyllids. The gene was expressed in both plants and insects infected with LsoA or LsoB. The highest relative expression was measured in LsoB-infected insects (Fig. 5). A two-way ANOVA was carried out on the relative expression of Lso-HPE1 by host type and ' $\mathrm{Ca}$. L. solanacearum' haplotype. There was a statistically significant interaction between the effects of the host type and the haplotype $(F[1,8]=24.66, P=0.001)$. Tukey's honestly significant difference posthoc tests determined that Lso-HPE1 relative expression was significantly higher in LsoBinfected psyllids compared with LsoA-infected psyllids $(P=$ $0.009)$ and LsoB-infected tomato $(P=0.006)$.

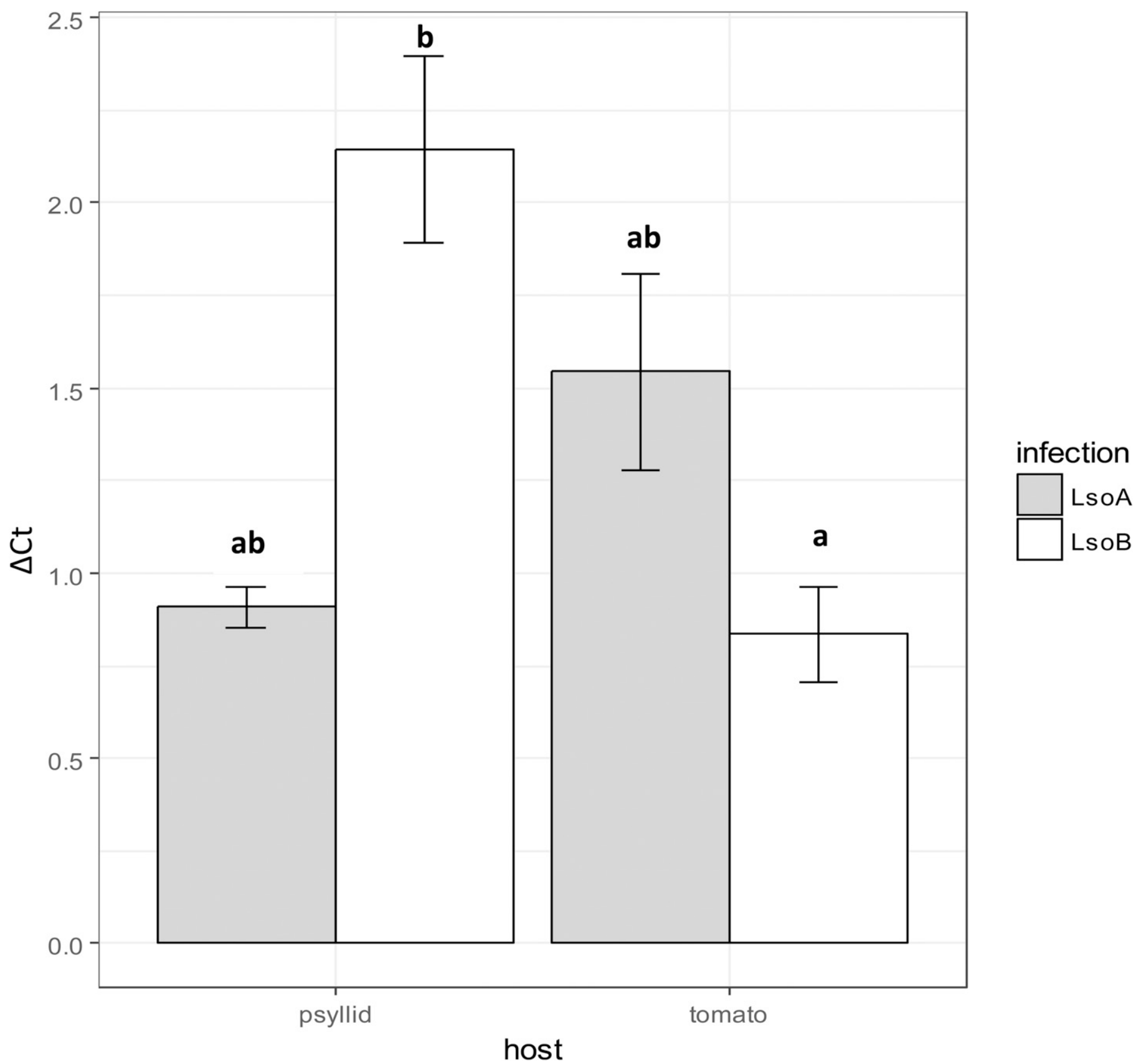

Fig. 5. Relative expression of 'Candidatus Liberibacter solanacearum' (Lso)-hypothetical protein effector 1 in LsoA- and LsoB-infected tomato plants and psyllids. Gene expression was quantified using reverse-transcription quantitative PCR and analyzed using the $\Delta$ threshold cycle $(\Delta \mathrm{Ct}) \mathrm{method}$. mRNA expression level was normalized relative to ' $\mathrm{Ca}$. $\mathrm{L}$. solanacearum' RecA. Bars represent mean $\Delta \mathrm{Ct} \pm$ standard error of the mean $(n=3)$. Letters indicate significant differences in gene expression as determined using two-way analysis of variance and Tukey's posthoc analyses. 


\section{DISCUSSION}

Despite the economic importance of diseases caused by ' $\mathrm{C} a$. L. solanacearum' and the expanding geographical range of these pathogens, very little is known about the molecular basis of their pathogenicity. Bacterial effectors able to suppress plant immune responses have been characterized (Jamir et al. 2004). Furthermore, Sec-translocon-dependent bacterial effectors were also shown to play a key role in disease development during infection with intracellular pathogens such as phytoplasmas (Hogenhout et al. 2009). Similarly to Liberibacter spp., phytoplasmas are also vectorborne, phloem-restricted, plant-pathogenic bacteria characterized by a reduced genome. Therefore, we hypothesize that Liberibacter spp. which encode a complete Sec secretion system (Ibanez et al. 2014) might also encode Sec-translocon-dependent proteins that play key roles in plant infection. Here, we have identified and characterized a Sec-translocon-dependent secreted ' $\mathrm{C} a$. L. solanacearum' protein which may play a role in suppressing proteintriggered cell death, part of the plant disease resistance response.

Findings from this study suggest that this protein is conserved among the ' $\mathrm{Ca}$. L. solanacearum' pathogens but not present in the other Liberibacter spp. The absence of Lso-HPE1 in two of the available LsoA genomes is probably due to the lower genome coverage of these assemblies. Indeed, the protein was found in the LsoB strain ZC1, the LsoA strains NZ1 and RSTM, as well as in the LsoC and LsoD genomes, in which it shows a high degree of similarity. Remarkably, a 23-amino-acid-long motif in the Cterminal region of the protein presents higher conservation between LsoA and LsoB than among other Lso-HPE1 proteins. We could speculate that this motif might be of interest for the protein activity, and could be associated with host or vector specificity. Indeed, these differences correlate with the geographic location and host association of these pathogens: ' $C a$. L. solanacearum' haplotypes $\mathrm{C}$ and $\mathrm{D}$ are associated with apiaceous crops in Europe, while haplotypes A and B are associated with solanaceous crops in North America (Haapalainen 2014). With only $43.58 \%$ identity, the most similar ' $C a$. L. asiaticus' protein is probably not a homolog protein. However, this ' $\mathrm{Ca}$. L. asiaticus' protein, which lacks the C-terminal domain that differentiate Lso-HPE1 proteins, is predicted to be secreted by the Sec-translocon system (Prasad et al. 2016). The cellular localization of this protein was shown to be temperature dependent in Agrobacterium-mediated transient expression assays in $N$. benthamiana epidermal cells (Liu et al. 2019).

SignalP predicted a 20 -amino-acid-long cleaved SP but this SP did not have the structure of other SEC targeting signals (CranfordSmith and Huber 2018). However, proteins lacking this type of SP can be recognized by the Sec machinery. Therefore, it was important to validate whether the predicted SP was active. The bioassay performed with the 25 amino acids of the Lso-HPE1 protein $\mathrm{N}$ terminus demonstrated a very strong secretion when compared with other secretion screening studies; in particular, the ones performed with ' $\mathrm{Ca}$. L. asiaticus' proteins (Prasad et al. 2016).

To examine the role of Lso-HPE1 in plant immunity, the proteins encoded by LsoA and LsoB were transiently expressed in $N$. benthamiana leaves. Neither of the Lso-HPE1 proteins triggered observable cell death when expressed alone, while they both were able to suppress cell death induced by two elicitors Bax and PrfD1416V in coinfiltration assays. These results led us to hypothesize that Lso-HPE1 can play a role in inhibiting immune responses similarly to AvrPto, a well-characterized bacterial effector involved in disrupting plant responses. In the present experiments, coinfiltration of Prf ${ }^{\mathrm{D} 1416 \mathrm{~V}}$ and Lso-HPE1 resulted in fewer cases of cell death compared with the infiltration of $\operatorname{Prf}^{\mathrm{D} 1416 \mathrm{~V}}$ alone or coinfiltration with the empty vector. Because Lso-HPE1 was able to disrupt the HR induced by $\operatorname{Prf}^{\mathrm{D} 1416 \mathrm{~V}}$, an autoactive variant of the Prf NBS-LRR resistance protein, this bacterial protein appears to interfere with ETI; thus, Lso-HPE1 could act in the signaling pathway downstream of the activation of $\mathrm{R}$ receptors or during the development of the cell death phenotype. The mechanisms involved in Bax-induced cell death in plants remain unknown. Bax is a mammal proapoptotic protein that is able to induce cell death in plants and yeast (Lacomme and Santa Cruz 1999; Zha et al. 1996). However, because Lso-HPE1 was able to suppress Bax-induced cell death, we speculate that this protein might act as a general cell death suppressor. The use of these two cell death inducers can help in deciphering the molecular role that bacterial effectors play in plants. For instance, potato cyst nematode effector RHA1B is able to suppress PrfD1416V - but not Bax-induced cell death (Kud et al. 2019), whereas the well-characterized Pseudomonas effector AvrPto is able to suppress the cell death induced by both proteins.

Bax induces cell death in a wide range of organisms; therefore, the ability of Lso-HPE1 to suppress Bax-induced cell death could imply that this bacterial protein is also able to suppress cell death in its vector, the potato psyllid. Indeed, programmed cell death is a common defense mechanism in insects (Hillyer 2016). However, apoptosis is not induced in the midgut of potato psyllids by ' $\mathrm{Ca}$. L. solanacearum' (Tang and Tamborindeguy 2019). This lack of apoptosis could result from the activity of Lso-HPE1 or other ' $\mathrm{Ca}$. L. solanacearum' effectors. Thus, it appears that testing the ability of putative bacterial effectors to suppress Bax-induced cell death in $N$. benthamiana could be used as a first screening method to identify candidate proteins involved in the interaction between plant pathogens and their vectors. Vectors of plant pathogens are usually hemipterans, for which genetic tools are sorely lacking.

Finally, because the expression of bacterial effectors are usually expressed during infection, we evaluated the expression of the LsoHPE1 in the vector and in tomato. We detected a higher expression of Lso-HPE1 in LsoB-infected insects compared with LsoA-infected insects and LsoB-infected plants. However, we found a higher but not significant relative expression of Lso-HPE1 in LsoA-infected tomato plants than LsoB-infected plants. The expression of this gene in insects supports the hypothesis that its role might include modulating the vector immune response. It is possible that Lso-HPE1 has a differential active role in insects and in plants. Further experiments are needed to identify the host proteins that interact with Lso-HPE1.

\section{ACKNOWLEDGMENTS}

We thank K. Swisher and J. Munyaneza (USDA-ARS, Wapato, WA) for supplying 'Ca. L. solanacearum'-infected psyllid DNA, S. Rahman and A. Azad (University of Maryland School of Medicine) for sharing the plasmid pJDT for the PhoA assay, and M. B. Dickman for providing pCXSN-Bax. J. G. Levy thanks E. A. Pierson.

\section{LITERATURE CITED}

Alfaro-Fernández, A., Siverio, F., Cebrián, M. C., Villaescusa, F. J., and Font, M. I. 2012. 'Candidatus Liberibacter solanacearum' associated with Bactericera trigonica-affected carrots in the Canary Islands. Plant Dis. 96:581.

Almagro Armenteros, J. J., Tsirigos, K. D., Sønderby, C. K., Petersen, T. N., Winther, O., Brunak, S., von Heijne, G., and Nielsen, H. 2019. SignalP 5.0 improves signal peptide predictions using deep neural networks. Nat. Biotechnol. 37:420-423.

Ammerman, N., Sayeedur Rahman, M., and Azad, A. F. 2008. Characterization of sec-translocon-dependent extracytoplasmic proteins of Rickettsia typhi. J. Bacteriol. 190:6234-6242.

Clark, K., Franco, J. Y., Schwizer, S., Pang, Z., Hawara, E., Liebrand, T. W. H., Pagliaccia, D., Zeng, L., Gurung, F. B., Wang, P., Shi, J., Wang, Y., Ancona, V., van der Hoorn, R. A. L., Wang, N., Coaker, G., and Ma, W. 2018. An effector from the Huanglongbing-associated pathogen targets citrus proteases. Nat. Commun. 9:1718.

Cranford-Smith, T., and Huber, D. 2018. The way is the goal: How SecA transports proteins across the cytoplasmic membrane in bacteria. FEMS Microbiol. Lett. 365:fny093.

Crosslin, J. M., Munyaneza, J. E., Brown, J. K., and Liefting, L. W. 2010. Potato zebra chip disease: A phytopathological tale. Plant Health Prog. 11.

DeYoung, B. J., and Innes, R. W. 2006. Plant NBS-LRR proteins in pathogen sensing and host defense. Nat. Immunol. 7:1243-1249. 
Du, X., Miao, M., Ma, X., Liu, Y., Kuhl, J. C., Martin, G. B., and Xiao, F. 2012. Plant programmed cell death caused by an autoactive form of Prf is suppressed by co-expression of the Prf LRR domain. Mol. Plant 5: 1058-1067.

Glynn, J. M., Islam, M., Bai, Y., Lan, S., Wen, A., Gudmestad, N. C., Civerolo, E. L., and Lin, H. 2012. Multilocus sequence typing of 'Candidatus Liberibacter solanacearum' isolates from North America and New Zealand. J. Plant Pathol. 94:223-228.

Greenway, G. A., and Rondon, S. 2018. Economic impacts of zebra chip in Idaho, Oregon, and Washington. Am. J. Potato Res. 95:362-367.

Haapalainen, M. 2014. Biology and epidemics of Candidatus Liberibacter species, psyllid-transmitted plant-pathogenic bacteria. Ann. Appl. Biol. 165:172-198.

Haapalainen, M., Latvala, S., Rastas, M., Wang, J., Hannukkala, A., Pirhonen, M., and Nissinen, A. I. 2018. Carrot pathogen 'Candidatus Liberibacter solanacearum' haplotype $\mathrm{C}$ detected in symptomless potato plants in Finland. Potato Res. 61:31-50.

Harrison, K., Tamborindeguy, C., Scheuring, D. C., Herrera, A. M., Silva, A., Badillo-Vargas, I. E., Miller, J. C., and Levy, J. G. 2019. Differences in zebra chip severity between 'Candidatus Liberibacter solanacearum' haplotypes in Texas. Am. J. Potato Res. 96:86-93.

Hillyer, J. F. 2016. Insect immunology and hematopoiesis. Dev. Comp. Immunol. 58:102-118.

Hogenhout, S. A., Van der Hoorn, R. A. L., Terauchi, R., and Kamoun, S. 2009. Emerging concepts in effector biology of plant-associated organisms. Mol. Plant-Microbe Interact. 22:115-122.

Ibanez, F., Levy, J., and Tamborindeguy, C. 2014. Transcriptome analysis of "Candidatus Liberibacter solanacearum" in Its psyllid vector, Bactericera cockerelli. PLoS One 9:e100955.

Ibanez, F., Levy, J., and Tamborindeguy, C. 2017. Identification and expression analyses of vitellogenin in Bactericera cockerelli (Sulc). J. Insect Physiol. 98:205-213.

Jain, M., Munoz-Bodnar, A., Zhang, S., and Gabriel, D. W. 2018. A secreted 'Candidatus Liberibacter asiaticus' peroxiredoxin simultaneously suppresses both localized and systemic innate immune responses in planta. Mol. Plant-Microbe Interact. 31:1312-1322.

Jamir, Y., Guo, M., Oh, H.-S., Petnicki-Ocwieja, T., Chen, S., Tang, X., Dickman, M. B., Collmer, A., and Alfano, J. R. 2004. Identification of Pseudomonas syringae type III effectors that can suppress programmed cell death in plants and yeast. Plant J. 37:554-565.

Jones, J. D., and Dangl, J. L. 2006. The plant immune system. Nature 444: 323-329.

Kud, J., Wang, W., Gross, R., Fan, Y., Huang, L., Yuan, Y., Gray, A., Duarte, A., Kuhl, J. C., Caplan, A., Goverse, A., Liu, Y., Dandurand, L.-M., and Xiao, F. 2019. The potato cyst nematode effector RHA1B is a ubiquitin ligase and uses two distinct mechanisms to suppress plant immune signaling. PLoS Pathog. 15:e1007720.

Lacomme, C., and Santa Cruz, S. 1999. Bax-induced cell death in tobacco is similar to the hypersensitive response. Proc. Natl. Acad. Sci. U.S.A. 96: 7956-7961.

Levy, J., Ravindran, A., Gross, D., Tamborindeguy, C., and Pierson, E. 2011. Translocation of 'Candidatus Liberibacter solanacearum', the zebra chip pathogen, in potato and tomato. Phytopathology 101:1285-1291.

Liefting, L. W., Perez-Egusquiza, Z. C., Clover, G. R., and Anderson, J. A. D. 2008. A new 'Candidatus Liberibacter' species in Solanum tuberosum in New Zealand. Plant Dis. 92:1474.

Liefting, L. W., Southerland, P. W., Ward, L. I., Paice, K. L., Weir, B. S., and Clover, G. R. G. 2009. A new "Candidatus Liberibacter" species associated with diseases of solanaceous crops. Plant Dis. 93:208-214.

Lin, H., Islam, M., Bai, Y., Wen, A., Lan, S., Gudmestad, N., and Civerolo, E. 2012. Genetic diversity of 'Candidatus Liberibacter solanacearum' strains in the United States and Mexico revealed by simple sequence repeat markers. Eur. J. Plant Pathol. 132:297-308.

Liu, X., Fan, Y., Zhang, C., Dai, M., Wang, X., and Li, W. 2019. Nuclear import of a secreted "Candidatus Liberibacter asiaticus" protein is temperature dependent and contributes to pathogenicity in Nicotiana benthamiana. Front. Microbiol. 10:1684.

Macho, A. P., and Zipfel, C. 2015. Targeting of plant pattern recognition receptor-triggered immunity by bacterial type-III secretion system effectors. Curr. Opin. Microbiol. 23:14-22.

Mendoza-Herrera, A., Levy, J., Harrison, K., Yao, J., Ibanez, F., and Tamborindeguy, C. 2018. Infection by 'Candidatus Liberibacter solanacearum' haplotypes A and B in Solanum lycopersicum 'Moneymaker'. Plant Dis. 102: 2009-2015.

Munyaneza, J. E., Crosslin, J. M., and Upton, J. E. 2007. Association of Bactericera cockerelli (Homoptera: Psyllidae) with "zebra chip," a new potato disease in southwestern United States and Mexico. J. Econ. Entomol. 100:656-663.

Nachappa, P., Levy, J., Pierson, E., and Tamborindeguy, C. 2011. Diversity of endosymbionts in the potato psyllid, Bactericera cockerelli (Hemiptera: Triozidae), vector of zebra chip disease of potato. Curr. Microbiol. 62: 1510-1520.

Nachappa, P., Levy, J., Pierson, E., and Tamborindeguy, C. 2014. Correlation between "Candidatus Liberibacter solanacearum" infection levels and fecundity in its psyllid vector. J. Invertebr. Pathol. 115:55-61.

Nelson, W. R., Fisher, T. W., and Munyaneza, J. E. 2011. Haplotypes of "Candidatus Liberibacter solanacearum" suggest long-standing separation. Eur. J. Plant Pathol. 130:5-12.

Pitino, M., Armstrong, C. M., Cano, L. M., and Duan, Y. 2016. Transient expression of Candidatus Liberibacter asiaticus effector induces cell death in Nicotiana benthamiana. Front. Plant Sci. 7:982.

Prasad, S., Xu, J., Zhang, Y., and Wang, N. 2016. SEC-translocon dependent extracytoplasmic proteins of Candidatus Liberibacter asiaticus. Front. Microbiol. 7:1989.

Ravindran, A., Saenkham, P., Levy, J. G., Tamborindeguy, C., Lin, H., Gross, D., and Pierson, E. A. 2018. Characterization of the serralysin-like gene of 'Ca. Liberibacter solanacearum' associated with potato zebra chip disease. Phytopathology 108:327-335.

Rojas-Martinez, R. I., Zavaleta-Mejia, E., Ochoa-Martinez, D. L., Alanis-Martines, I., and Garcia-Tapia, F. 2016. Association of Candidatus Liberibacter solanacearum with the decline of tomato (Solanum lycopersicum L.). J. Plant Pathol. 98:191-196.

Ronquist, F., Teslenko, M., Van Der Mark, P., Ayres, D. L., Darling, A., Höhna, S., Larget, B., Liu, L., Suchard, M. A., and Huelsenbeck, J. P. 2012. MrBayes 3.2: Efficient Bayesian phylogenetic inference and model choice across a large model space. Syst. Biol. 61:539-542.

Salmeron, J. M., Oldroyd, G. E., Rommens, C. M., Scofield, S. R., Kim, H. S., Lavelle, D. T., Dahlbeck, D., and Staskawicz, B. J. 1996. Tomato Prf is a member of the leucine-rich repeat class of plant disease resistance genes and lies embedded within the Pto kinase gene cluster. Cell 86:123-133.

Secor, G. A., and Rivera-Varas, V. V. 2004. Emerging diseases of cultivated potato and their impact on Latin America. Rev. Latinoam. Papa 1:1-8.

Swisher Grimm, K. D., and Garczynski, S. F. 2019. Identification of a new haplotype of 'Candidatus Liberibacter solanacearum' in Solanum tuberosum. Plant Dis. 103:468-474.

Swisher Grimm, K. D., Mustafa, T., Rodney Cooper, W., and Munyaneza, J. E. 2018. Role of 'Candidatus Liberibacter solanacearum' and Bactericera cockerelli haplotypes in zebra chip Incidence and symptom severity. Am. J. Potato Res. 95:709-719.

Tahzima, R., Maes, M., Achbani, E. H., Swisher, K. D., Munyaneza, J. E., and De Jonghe, K. 2014. First report of 'Candidatus Liberibacter solanacearum' on carrot in Africa. Plant Dis. 98:1426.

Tamborindeguy, C., Huot, O. B., Ibanez, F., and Levy, J. 2017. The influence of bacteria on multi-trophic interactions among plants, psyllids, and pathogen. Insect Sci. 24:961-974.

Tamura, K., Peterson, D., Peterson, N., Stecher, G., Nei, M., and Kumar, S. 2011. MEGA5: Molecular evolutionary genetics analysis using maximum likelihood, evolutionary distance, and maximum parsimony methods. Mol. Biol. Evol. 28:2731-2739.

Tang, X.-T., and Tamborindeguy, C. 2019. No evidence of apoptotic response of the potato psyllid, Bactericera cockerelli, to "Candidatus Liberibacter solanacearum" at the gut interface. Infect. Immun. e00242-00219. doi: 10.1128/IAI.00242-19

Teresani, G. R., Bertolini, E., Alfaro-Fernández, A., Martínez, C., Tanaka, F. A. O., Kitajima, E. W., Roselló, M., Sanjuán, S., Ferrándiz, J. C., López, M. M., Cambra, M., and Font, M. I. 2014. Association of 'Candidatus Liberibacter solanacearum' with a vegetative disorder of celery in Spain and development of a Real-Time PCR method for its detection. Phytopathology 104:804-811.

Toruño, T. Y., Stergiopoulos, I., and Coaker, G. 2016. Plant-pathogen effectors: Cellular probes interfering with plant defenses in spatial and temporal manners. Annu. Rev. Phytopathol. 54:419-441.

Xiang, J., Li, X., Wu, J., Yin, L., Zhang, Y., and Lu, J. 2016. Studying the mechanism of Plasmopara viticola RxLR effectors on suppressing plant immunity. Front. Microbiol. 7:709.

Yao, J., Saenkham, P., Levy, J., Ibanez, F., Noroy, C., Mendoza, A., Huot, O., Meyer, D. F., and Tamborindeguy, C. 2016. Interactions 'Candidatus Liberibacter solanacearum'-Bactericera cockerelli: Haplotype effect on vector fitness and gene expression analyses. Front. Cell. Infect. Microbiol. 6:62.

Zha, H., Fisk, H. A., Yaffe, M. P., Mahajan, N., Herman, B., and Reed, J. C. 1996. Structure-function comparisons of the proapoptotic protein Bax in yeast and mammalian cells. Mol. Cell. Biol. 16:6494-6508. 